\title{
Disseminated Double-Hit Lymphoma in a Young Adult
}

Karen Damian and Jonathan Emmanuel Cancio

Department of Laboratories, University of the Philippines-Philippine General Hospital

\section{ABSTRACT}

High-grade B-cell lymphoma with MYC and BCL2 and-or BCL6 rearrangements, also called doublehit lymphoma, is an aggressive mature B-cell lymphoma that carries both MYC and BCL2 and/or BCL6 translocations. This can present as a diagnostic challenge since clinical characteristics, morphology and immunophenotype are not accurate indicators of underlying genetic aberrations in this type of lymphoma. We report a case of a 25 -year-old male who presented with a one-year history of cough, gradually increasing abdominal girth and jaundice. Definitive diagnosis was made post-mortem with additional ancillary studies using immunohistochemistry staining and fluorescent in-situ hybridization studies.

Key words: lymphoma, B-cell lymphoma, molecular diagnostic technique

ISSN 2507-8364 (Online)

Printed in the Philippines.

Copyright $\odot 2021$ by the PJP.

Received: 6 May 2021.

Accepted: 30 May 2021.

Published online first: 30 June 2021

https://doi.org/10.21141/PJP.2021.07

Corresponding author: Karen B. Damian, $M D$

E-mail:ksbuselcodamian@up.edu.ph

\section{INTRODUCTION}

In 2016, the World Health Organization (WHO) established a new criterion under the high-grade B-cell lymphoma which describes a tumor with a unique molecular pathogenesis. This lymphoma involves the presence of translocations in the MYC gene and BCL-2 or less frequently the BCL-6 genes, hence the name "double hit lymphoma" (DHL). ${ }^{1}$ The diagnosis of DHL requires molecular techniques, most commonly by fluorescence is situ hybridization (FISH), without much regard for its clinical features and heterogenous morphologies. Double hit lymphomas confer poorer prognosis and lower response to usual chemotherapy regimens. ${ }^{1}$ Here we present an autopsy case of a 25-year-old male who presented with one year history of cough, increasing abdominal girth, jaundice and abdominal pain.

\section{CASE}

The patient was a 25-year-old, male admitted for abdominal pain. Two years prior to admission, patient had repeated episodes of non-productive cough with accompanying undocumented, on and off fever. One year prior to admission, patient had more frequent febrile episodes still with non-productive cough. Over time, he began experiencing recurrent burning epigastric pain with increasing abdominal girth, early satiety, generalized weakness, progressive weight loss, bipedal edema and jaundice. Patient was eventually admitted at our institution with physical examination findings showing tachycardia, tachypnea, icteric sclerae, decreased breath sounds, a distended abdomen with right hemiabdominal tenderness, jaundice, and bipedal edema. Laboratory exams revealed anemia, thrombocytopenia, deranged coagulation parameters, hypercalcemia, hypoalbuminemia, elevated transaminases, elevated alkaline phosphatase, three-fold elevation in CA 19-9 and a non-reactive hepatitis profile. Radiographic studies revealed pulmonary changes and bilateral pleural effusion. Ultrasound findings of the liver revealed multiple hypoechoic nodules, largest at segment VIII measuring $3 \times 3 \times 6 \mathrm{~cm}$ with non-dilated intrahepatic ducts and common bile duct. Ascites was 
minimal. Patient's condition deteriorated over the course of admission with persistent fever, progressing jaundice and anasarca. Biopsy of the liver nodules was not done due to the unstable condition of the patient. Despite medical management and intubation, patient succumbed on his ninth hospital day of admission.

\section{AUTOPSY FINDINGS}

The decedent was received for partial autopsy 7-hours postmortem. The head was normocephalic with note of facial edema, particularly evident at the periorbital area and lips. The sclerae were icteric. The neck was symmetrical with multiple palpable lymph nodes. The abdomen was soft and distended with a measured girth of $89 \mathrm{~cm}$. There were multiple petechiae noted over the anterior abdomen.

Upon opening of the thoraco-abdominal area, the liver was studded with multiple, yellow, firm lesions. Strawcolored, ascitic fluid amounting to $1600 \mathrm{cc}$ was drained. Bilateral serosanguinous pleural fluid amounting to $400 \mathrm{cc}$ on the right and $360 \mathrm{cc}$ on the left were also drained. The liver was enlarged, weighing 2,700 grams, well-above the normal reference range. Its surface was nodular and studded with multiple yellow, firm masses, the largest measuring $9 \mathrm{~cm}$ in greatest diameter. Cut sections revealed a red-brown parenchyma with yellow, firm nodules interspersed throughout the substance of the liver (Figure 1A). Microscopic sections of the liver revealed sheets of hyperchromatic cells with a diffuse growth pattern. On higher magnification, the cells were atypical and pleomorphic with abundant cytoplasm, large, hyperchromatic nuclei and prominent nucleoli (Figure 1B-C). Mitotic figures and apoptotic cells were also identified. The spleen was also enlarged, weighing 285 grams, above the normal reference range. The capsule was intact with multiple, yellow, firm nodules throughout. Cut sections showed multiple, yellow, firm nodules scattered throughout the entirety of the splenic parenchyma. Microscopic sections revealed similar tumor infiltrates as that of the liver (Figure 2A). The pancreas, gallbladder, bilateral kidneys, bilateral adrenal glands, bilateral lungs all showed variably-sized nodules of the same gross and tumor microscopic features as that of the liver and spleen. The bone marrow smears revealed atypical lymphoid population with large irregular nuclei, prominent nucleoli and moderate cytoplasm (Figure 2B). Enlarged pulmonary hilar lymph nodes with sizes ranging from 3 to $8 \mathrm{~cm}$ in greatest diameter were also noted on further dissection, cut sections of which showed tanbrown, fleshy to friable surfaces. Enlarged lymph nodes were also noted in the peripancreatic and inguinal areas. Microscopic examination revealed effacement of the usual lymph node architecture and replaced by sheets of atypical, pleomorphic, discohesive cells with large nucleus and prominent nucleolus, some cells exhibiting eosinophilic nucleoli (Figure 2C-D). Immunohistochemistry studies were done on the posterior mediastinal lymph node which revealed positivity for the following stains: Leukocyte common antigen (LCA), CD20 (diffuse, strong), PAX5 (diffuse, strong), CD30 (patchy, weak) (Figure 3). Subsequent immunohistochemistry studies were done which revealed positivity for BCL-2 (>40\%), C-MYC
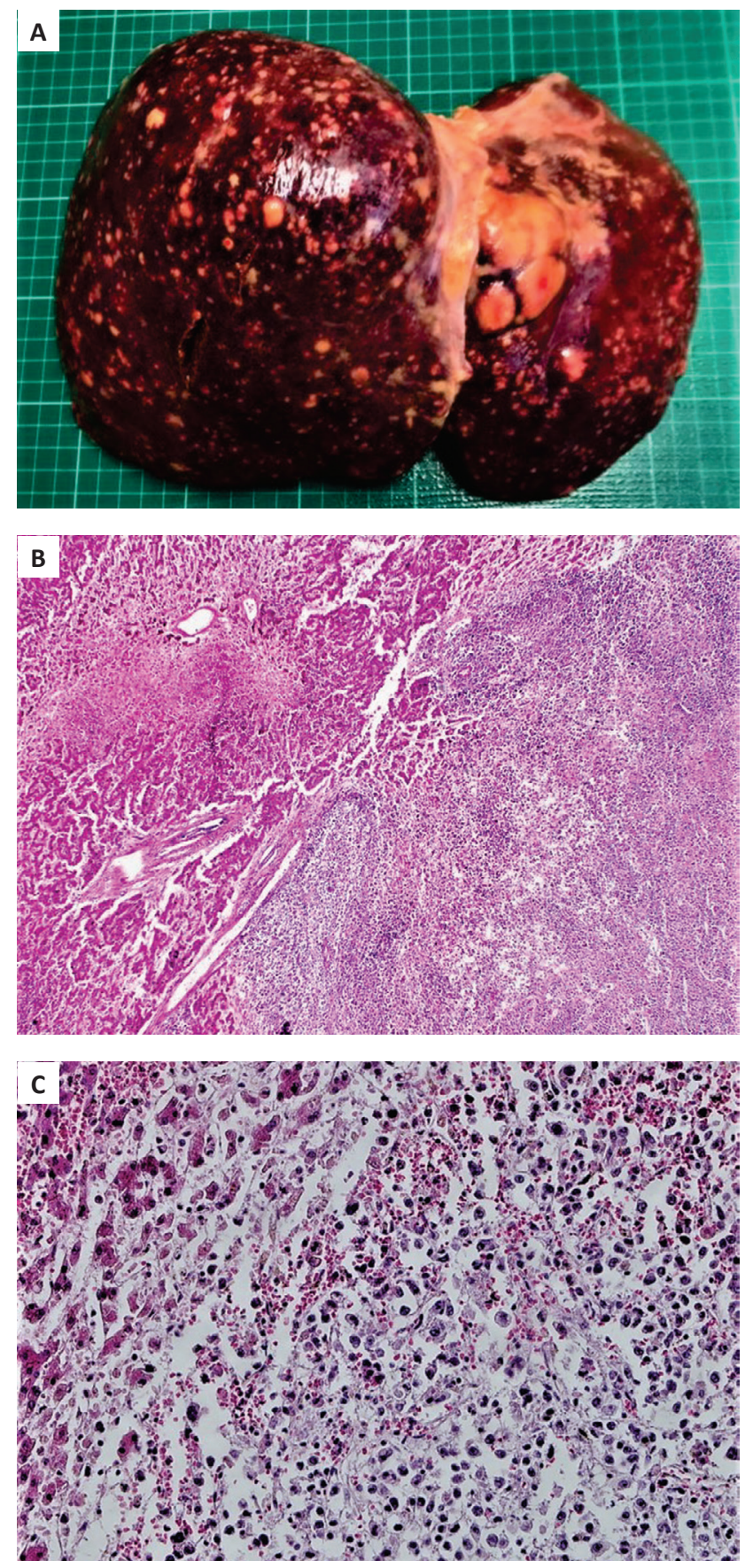

Figure 1. (A) Gross appearance of the liver showing variablysized, firm, nodules throughout; (B) Photomicrographs of the liver showing sheets of tumor infiltrates within the parenchyma, H\&E, 10x and (C) 40x.

$(>50 \%)$ and a proliferative index of 50-60\% using Ki-67. The tumor cells were negative for the following stains: CD5, TdT, Cyclin D1, CD10, BCL-6, MUM-1, pancytokeratin and HMB-45. Molecular analysis using FISH was done revealing translocation rearrangements in MYC (5.56\%) and BCL-2 (8.25\%), trisomy 8 (IgH/Myc fusion), and no BCL-6 rearrangement (Figure 4).

Given the said morphology, the immunophenotype and molecular characteristics, the final diagnosis given to this case was a high-grade B-cell lymphoma with MYC and BCL-2 rearrangements (double hit lymphoma). 

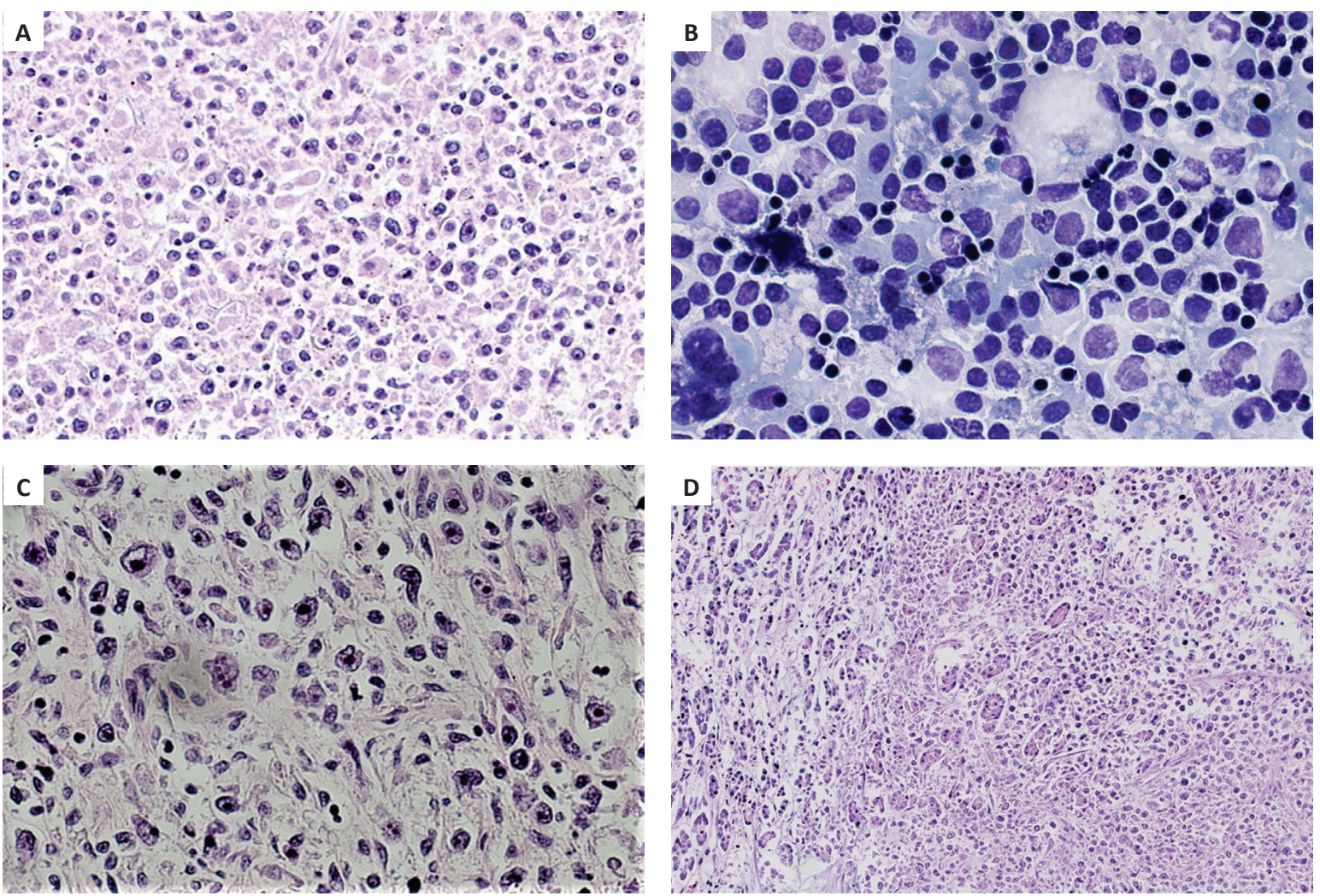

Figure 2. (A) Photomicrographs of the tumor infiltrates in the spleen, H\&E, 40x; (B) bone marrow, Wright, 100x; (C) perihilar lymph node, H\&E, 40x and (D) pancreas, H\&E, 40x. The tumor cells show large, vesicular nuclei with prominent nucleoli and scant cytoplasm.
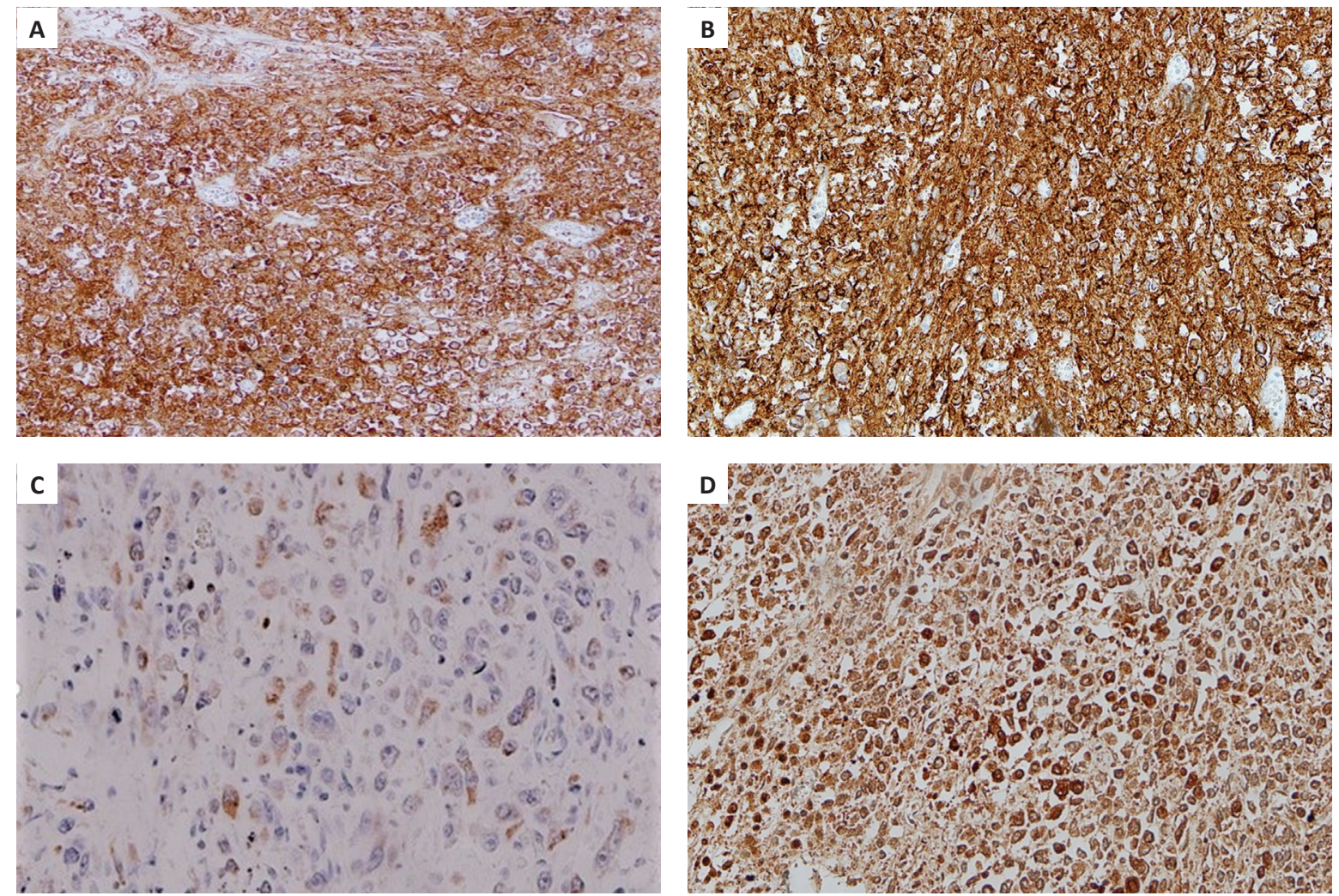

Figure 3. (A) Neoplastic cells showing positivity with the following immunohistochemistry studies: LCA, 40x; (B) CD20, 40x; (C) bcl-2, 40x; (D) and C-myc, 40X. LCA, CD 20 and C-myc show diffuse, strong positivity while bcl-2 showed variable staining intensity in $>40 \%$ of the tumor cells. 

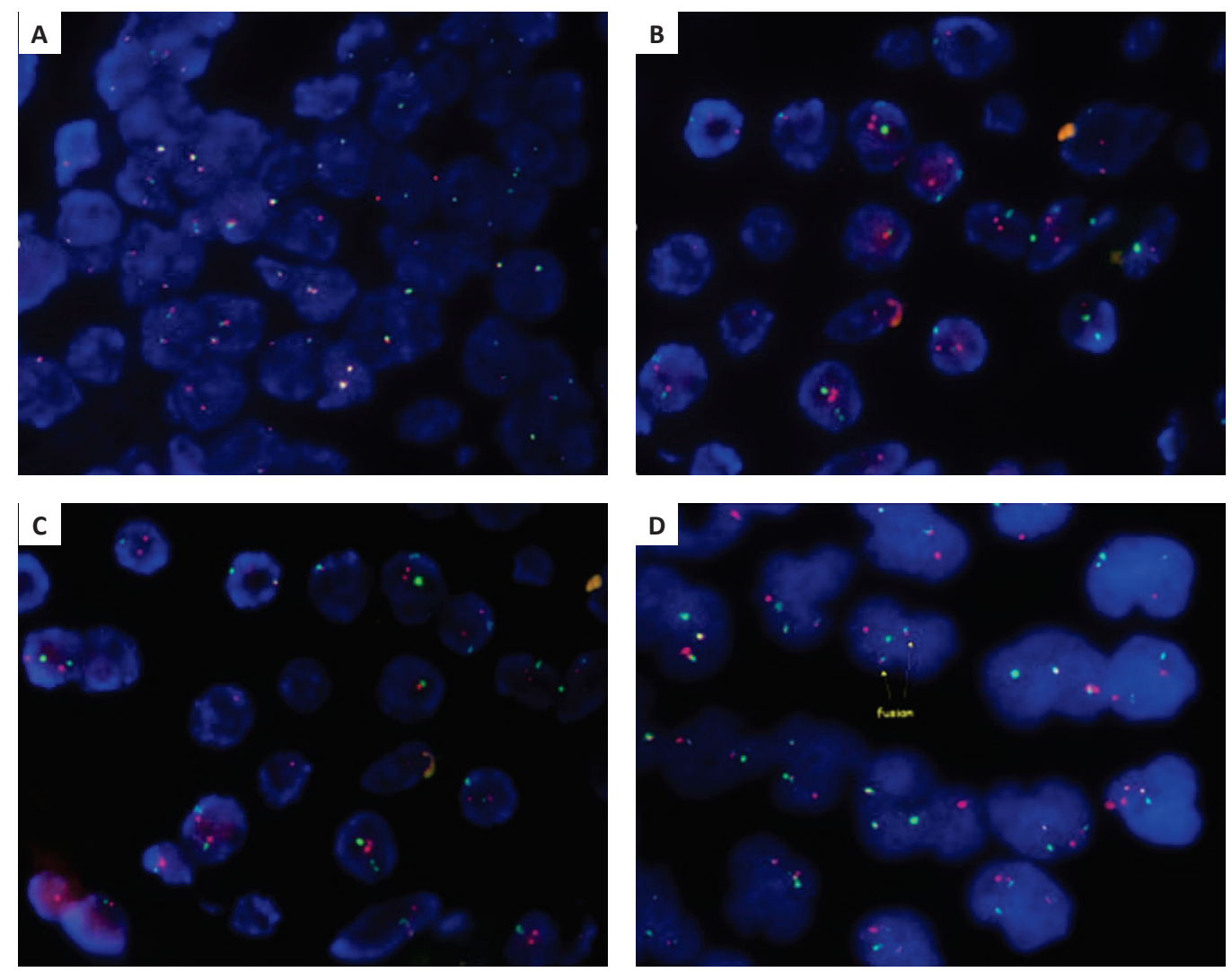

Figure 4. (A) Fluorescent in-situ hybridization study showing: Absence of BCL-6 rearrangement; (B) presence of MYC rearrangement (11.11\%, cut-off value of $>10 \%)$; (C) BCL-2 rearrangement $(8.25 \%$, cut-off value of $>8 \%)$; (D) and, $t(8: 14)$ aberration on the MYC gene (8q24) (5.66\%, cut-off value of $2 \%)$.

\section{DISCUSSION}

With the advent of more discoveries about the biology and genetics of hematolymphoid neoplasms, newer types of lymphomas have been identified and reclassified by the WHO. ${ }^{1,2}$ Among these lymphomas are the so-called double-hit lymphomas. The term "double hit" as defined for this category refers only to the co-occurrence of MYC and BCL-2 and/or BCL-6 translocations. Exceptions for this category are lymphomas that do not harbor MYC translocations as well as MYC-associated translocations in other genes other than BCL-2 and/or BCL-6, transformed follicular lymphomas and B-lymphoblastic leukemia/ lymphoma with MYC and BLC-2 translocations. ${ }^{1}$ Much interest has been piqued by aggressive B-cell lymphomas, particularly DLBCLs, because a small subgroup of these patients were observed to be resistant to standard chemotherapy, had dismal overall survival despite treatment and preponderance of extranodal spread particularly in the central nervous system and bone marrow. ${ }^{1-3}$ The advances in molecular testing has paved the way for the identification of certain genetic aberrations that have led to the establishment of this new category of aggressive B-cell lymphoma. ${ }^{3}$ Diagnosis is made by detection of rearrangements of MYC, BCL-2, and BCL-6 by a cytogenetic or molecular method such as FISH.

A peculiarity in our case is the age at presentation of the patient. Majority of double-hit lymphoma cases present in elderly patients, with a median age at diagnosis ranging from 51-65 years, and a slight male predominance. ${ }^{1}$ DHL are reported rarely in young adults, adolescents and children. ${ }^{4,5}$ To date, there has been no reported case of DHL among the said population in the Philippines.

Most of the patients were diagnosed in the advanced stages and presented with widespread disease, with most cases involving the central nervous system (CNS) and bone marrow. A study by Niitsu, et al., reported several cases of double hit lymphoma with pleural effusion, similar to our case. ${ }^{6}$ Niitsu also reported twelve cases which had three extranodal sites of involvement. A study by Guillermo et al., reported the distribution of disease according to nodal and extranodal sites and noted cases with liver involvement in only $2 \%$ of cases. He also reported only one case out of 382 with a double extranodal site of involvement, that being the bilateral breasts and ovary. ${ }^{7} \mathrm{~A}$ literature search of multiorgan involvement by double hit lymphoma yielded no reports with more than three sites of involvement. To the best of our knowledge, no case has ever been reported with such extensive, widespread organ involvement such as ours. Presenting symptoms may be related to the rapidly enlarging mass depending upon the site of the mass; B symptoms such as fever, night sweats, weight loss may be seen in a number of patients. Our case was consistent with published reports, presenting with disseminated disease and accompanying signs and symptoms correlate to the sites of involvement. These include increasing abdominal girth, jaundice and anasarca due to hepatosplenomegaly and tachypnea due to pleural effusion and enlarging perihilar lymph nodes. Laboratory test results were also consistent with the autopsy findings, 
with most tests having deranged results due to hepatic and pancreatic involvement by tumor. It is unfortunate that the autopsy was only a partial one which precluded us from further evaluation of the CNS.

Double hit lymphomas have a heterogenous morphologic appearance, with most authors agreeing to a higher-grade type of morphology- DLBCL-like, blastoid and Burkittlike. Most of the reported cases appear to exhibit a DLBCL morphology with a diffuse growth pattern, variable nuclear size, prominence of nucleoli, variable mitotic figures and variable proliferation rate. ${ }^{1,3,8}$ Our case also reports similar high-grade, diffuse large B-cell lymphomalike morphologic features with that of previously reported cases.

Earlier studies have suggested the use of immunohistochemistry studies, particularly CD10, BCL-6, IRF4/ MUM-1, BCL-2 and C-MYC as a surrogate test to identify patients who are likely candidates for further FISH testing. The first three mentioned stains are utilized to determine cell of origin (COO) wherein DLBCLs are classified according to the stage of differentiation where the malignant cells are derived..$^{3,9,10}$ The most commonly used method to determine cell of origin is the Hans algorithm, a binary classifier, assigning the DLBCLs into either germinal center or non-germinal center phenotype. Most studies report that DHLs are of germinal center origin, particularly those with the MYC/BCL-2 translocation. However, a small percentage of DHLs, specifically those with the MYC/BCL-6 translocation will have a non-germinal center differentiation. It is for this reason that using cell of origin as a surrogate test to determine cases which should undergo FISH may not be the most ideal because a small percentage of cases will be missed..$^{3,9}$ Another frequently suggested method to predict cases with DHL is thru the use of BCL-2 and MYC immunohistochemistry studies. ${ }^{11-14}$ This comes from the premise that these two genes are overexpressed in DHL. However, more and more studies have shown that dual protein expression (DPE) among DLBCLs can occur without genetic aberrations. ${ }^{1-3,11-15}$ The WHO 2016 reports that although this is not currently accepted as a new, separate entity, DPE in DLBCLs can confer a poorer prognosis with worse survival outcome compared to other DLBCL subtypes. Several studies also caution the use of DPE to identify which cases should undergo further FISH testing citing differences in cutoffs as a reason that cases can be missed..$^{3,10,11-15}$ Our case had an immunohistochemistry result which showed DPE with C-MYC and BCL-2. Given the staining result coupled with the aggressive clinical history, this prompted us to do further testing with FISH to confirm the presence of chromosomal aberrations. Most authors recommend FISH analysis for all cases of DLBCLs. ${ }^{1,2}$ However, due to limited availability of this test as well as financial constraints from our patients, this will be very difficult to implement as a standard practice. Although selection by immunohistochemistry studies is not the most optimal way to determine which cases to FISH due to variable concordance, as reported by most authors, we opted to go this route given the above-mentioned limitations.

Double hit lymphomas have been given much attention of late due advancements in molecular pathology. Interest has been shown by both clinicians and pathologists alike because of its poor response to usual chemotherapeutic treatment protocols and overall poor survival. At present, no standard treatment protocol has been developed for this disease. ${ }^{16}$ Clinical trials are still underway to determine which may confer better progression free survival and overall survival.

\section{CONCLUSION}

We present a case of an aggressive B-cell lymphoma that had an unusual clinical presentation. This case affirms the fact that lymphoma is a great mimic and that it should always be one of the considerations especially when faced with a clinical diagnostic dilemma. Definitive diagnosis was made post-mortem which highlights the value of autopsy and exhaustive but cost-effective work-up. Awareness that this type of lymphoma exists is a foreground which prompted us to report this case. Together with a careful evaluation of clinical, morphologic and immunophenotypic predictors, we recommend a sequential approach to the diagnosis of double hit lymphomas especially in a low-resource setting such as ours.

\section{ACKNOWLEDGMENT}

The authors would like to acknowledge the National Kidney and Transplant Institute for granting permission to use photos of the FISH analysis of the patient.

\section{ETHICAL CONSIDERATION}

Patient consent was obtained before submission of the manuscript.

\section{STATEMENT OF AUTHORSHIP}

Both authors certified fulfillment of ICMJE authorship criteria.

\section{AUTHOR DISCLOSURE}

Both authors declared no conflict of interest.

\section{FUNDING SOURCE}

The FISH analysis of the case was generously funded by the Department of Internal Medicine, University of the Philippines - Philippine General Hospital.

\section{REFERENCES}

1. Swerdlow SH, Campo E, Harris NL, et al, eds. WHO classification of tumours of haematopoietic and lymphoid tissues, rev. 4th ed, vol. 2. Lyon, International Agency for Research on Cancer; 2017

2. Smith SM. Aggressive B-cell lymphoma: the double-hit and double-expresser phenotypes. Clin Adv Hematol Oncol. 2017;15(1):40-2. PMID: 28212368.

3. Stephens DM, Smith SM. Diffuse large B-cell lymphoma- who should we FISH? Ann Lymphoma. 2018; 2:1-10. https://doi.org/10.21037/aol.2018.11.01.

4. Aukema SM, Siebert R, Schuuring E, et al. Doublehit-B-cell lymphoma. Blood. 2011; 117(8):2319-31. 
PMID: 21119107. https://doi.org/10.1182/blood-201009-297879.

5. Msimang MZ, Ramdial PK, Kuppusamy JB, Nargan $\mathrm{K}$, Sheik-Gafoor MH. AIDS-associated pediatric high-grade B-cell lymphoma with MYC and BCL2 translocations. J AIDS Clin Res. 2018;8(11):742. https://doi.org/10.4172/2155-6113.1000742.

6. Niitsu N, Okamoto M, Miura I. et al. Clinical features and prognosis of de novo diffuse large B-cell lymphoma with $\mathrm{t}(14 ; 18)$ and $8 \mathrm{q} 24 / \mathrm{c}-\mathrm{MYC}$ translocations. Leukemia. 2009;23(4):777-83. PMID: 19151788. https://doi.org/10.1038/leu.2008.344.

7. López-Guillermo A, Colomo L, Jímenez M, et al. Diffuse large B-cell lymphoma: clinical and biological characterization and outcome according to the nodal or extranodal primary origin. J Clin Oncol. 2016; 23(12):2797-804. PMID: 15728226. https://doi.org/ 10.1200/JCO.2005.07.155.

8. Oliveira CC, Maciel-Guerra H, Kucko L, et al. Double-hit lymphomas: clinical, morphological, immunohistochemical and cytogenetic study in a series of Brazilian patients with high-grade nonHodgkin lymphoma. Diagn Pathol. 2017;12(1):3. PMID: 28061782. PMCID: PMC5219810. https://doi. org/10.1186/s13000-016-0593-0.

9. Friedberg, JW. How I treat double hit lymphoma. Blood. 2017;130(5): 590-6. PMID: 28600333. https:// doi.org/10.1182/blood-2017-04-737320.

10. Liu Y, Barta SK. Diffuse large B-cell lymphoma: 2019 update on diagnosis, risk stratification, and treatment. Am J Hematol. 2019;95(5):604-16. PMID: 30859597. https://doi.org/10.1002/ajh.25460.
11. Kim H, Kim HJ, Kim SH. Diagnostic approach for double-hit and triple-hit lymphoma based on immunophenotypic and cytogenetic characteristics of bone marrow specimens. Ann Lab Med. 2020;40(5): 361-9. PMID: 32311849. PMCID: PMC7169621. https://doi.org/10.3343/alm.2020.40.5.361.

12. Friedberg JW. Double hit diffuse large B-cell lymphomas: diagnostic and therapeutic challenges. Chin Clin Oncol. 2015; 4(1):9. PMID: 25841716. https://doi.org/10.3978/j.issn.2304-3865.2015.03.02.

13. Davies, A. Double-hit lymphoma: so what? Hematol Oncol. 2019; 37(Suppl 1):19-23. PMID: 31187528. https://doi.org/10.1002/hon.2581.

14. Di Napoli A, Remotti D, Agostinelli C, et al. A practical algorithmic approach to mature aggressive B cell lymphoma diagnosis in the double/triple hit era: selecting cases, matching clinical benefit. Virchows Archiv. 2019;475(4):513-8. PMID: 31388760. PMCID: PMC6828632. https://doi.org/10.1007/s00428-01902637-2.

15. Ambrosio MR, Lazzi S, Lo Bello G, et al. MYC protein expression scoring and its impact on the prognosis of aggressive B-cell lymphoma patients. Haematologica. 2019;104(1):e25-8. PMID: 29954940. PMCID: PMC6312021. https://doi.org/10.3324/ haematol.2018.195958.

16. Phuoc V, Sus J, Chavez JC. Drug therapy for doublehit lymphoma. Drugs Context. 2019;8:2019-8-1. PMID: 31844420. PMCID: PMC6905641. https://doi. org/10.7573/dic.2019-8-1.

Disclaimer: This journal is OPEN ACCESS, providing immediate access to its content on the principle that making research freely available to the public supports a greater global exchange of knowledge. As a requirement for submission to the PJP, all authors have accomplished an AUTHOR FORM, which declares that the ICMJE criteria for authorship have been met by each author listed, that the article represents original material, has not been published, accepted for publication in other journals, or concurrently submitted to other journals, and that all funding and conflicts of interest have been declared. Consent forms have been secured for the publication of information about patients or cases; otherwise, authors have declared that all means have been exhausted for securing consent.

\section{Publish in the new PJP. Visit our website: http://philippinejournalofpathology.org}

